\title{
On uniqueness and reconstruction of rough and complex obstacles from acoustic scattering data
}

\author{
Mourad Sini \\ RICAM, Austrian Academy of Sciences \\ 69 Altenbergerstrasse, A 4040, Linz, Austria. \\ E-mail: mourad.sini@oeaw.ac.at
}

\begin{abstract}
We deal with the inverse scattering problem by an obstacle at a fixed frequency. The obstacle is characterized by its shape, the type of boundary conditions on its surface and the eventual coefficients distributed on this surface. In this paper, we assume that the surface $\partial D$ of the obstacle $D$ is Lipschitz and the surface impedance, $\lambda$, is given by a complex valued, measurable and bounded function. We prove uniqueness of $(\partial D, \lambda)$ from the far field map under these regularity conditions. The usual proof of uniqueness for obstacles, based on the use of singular solutions, is divided into two steps. The first one consists of the use of Rellich type lemma to go from the far fields to the near fields and then use the singularities of the singular solutions, via orthogonality relations, to show uniqueness of $\partial D$. The second step is to use the boundary conditions to prove uniqueness of $\lambda$ on $\partial D$ via the unique continuation property. This last step requires the surface impedance to be continuous. We propose an approach using layer potentials to transform the inverse problem to the invertibility of integral equations of second kind involving the unknowns $\partial D$ and $\lambda$. This enables us to weaken the required regularity conditions by assuming $\partial D$ to be Lipschitz and $\lambda$ to be only bounded. The procedure of the proof is reconstructive and provides a method to compute the complex valued and bounded surface impedance $\lambda$ on $\partial D$ by inverting an invertible integral equation. In addition, assuming $\partial D$ to be $C^{2}$ regular and $\lambda$ to be of class $C^{0, \alpha}$, with $\alpha>0$, we give a direct formula as another method to reconstruct the surface impedance on $\partial D$.
\end{abstract}

2000 Mathematics Subject Classification: 35P25, 35R30, 45Q05, 78A45.

Keywords: Scattering by obstacles, farfield map, Helmholtz..

\section{Introduction and statement of the results}

Let $D$ be a bounded and a Lipschitz domain of $\mathbb{R}^{n}, n=2,3, \ldots$, such that $\mathbb{R}^{n} \backslash \bar{D}$ is connected. The propagation of time-harmonic acoustic fields in a homogeneous media is governed by the Helmholtz equation

$$
\Delta u+\kappa^{2} u=0 \quad \text { in } \quad \mathbb{R}^{n} \backslash \bar{D}
$$

where $\kappa$ is the real positive wave number. At the boundary of the scatterers the total field $u$ satisfies the impedance boundary condition

$$
\frac{\partial u}{\partial \nu}+i \lambda u=0 \text { on } \partial D
$$


with some measurable and bounded complex valued function $\lambda$ on $\partial D$ with positive real part where $\nu$ is the unit normal directed outside of $D$. Given an incident field $u^{i}$ which satisfies $\Delta u^{i}+\kappa^{2} u^{i}=0$ we look for solutions $u:=u^{i}+u^{s}$ of (1) and (2) where the scattered field $u^{s}$ is assumed to satisfy the Sommerfeld radiation condition

$$
\lim _{r \rightarrow \infty} r^{\frac{n-1}{2}}\left(\frac{\partial u^{s}}{\partial r}-i \kappa u^{s}\right)=0
$$

$r=|x|$ and the limit is uniform with respect to all the directions $\theta:=\frac{x}{|x|}$.

The forward scattering problem (1)-(2)-(3) with the mentioned regularity conditions on $(\partial D, \lambda)$ is well posed. There are two ways to justify it. The first one is by using the limiting absorption principle as it is done in ([25], section 4). The second one is the approach by Brakhage-Werner-Leis-Panic which consist of a combination of the single layer and double layer potentials. Indeed, this can be done following the arguments by Leis as explained in ([7], pages 92-93) and using the layer potential mappings stated on $C^{2}$-smooth domains, see [7] or on Lipschitz domains, see [10] and [22].

It is well known (see [8], [25]) that this reflected field satisfies the following asymptotic property,

$$
u^{s}(x)=\frac{e^{i \kappa r}}{r^{\frac{n-1}{2}}} u^{\infty}(\theta)+O\left(r^{-\frac{n+1}{2}}\right), \quad r \rightarrow \infty,
$$

where the function $u^{\infty}(\cdot)$ defined on the unit n-dimensional sphere $\mathbb{S}^{n}$ is called the far-field associated to the incident field $u^{i}$. Taking particular incident fields given by the plane waves, $u^{i}(x, d):=e^{i \kappa d \cdot x}, d \in \mathbb{S}^{n}$, we define the far-field pattern $u^{\infty}(\theta, d)$ for $(\theta, d) \in \mathbb{S}^{n} \times \mathbb{S}^{n}$. Analogously, for an incident point source $\Phi(\cdot, z)$, given by the fundamental solution of $\Delta+\kappa^{2}$ in $\mathbb{R}^{n}$,

$$
\Phi(x, y):=\frac{i \gamma_{n}}{|x-y|^{\frac{n-2}{2}}} H_{\frac{n-2}{2}}^{(1)}(\kappa|x-y|), x \neq y, x, y \in \mathbb{R}^{n}
$$

we denote the scattered field by $\Phi^{s}(\cdot, z)$. The function $H_{\frac{n-2}{2}}^{(1)}$ is the Hankel function of the first kind and of order $\frac{n-2}{2}$. The constant $\gamma_{n}$ is given by $\gamma_{n}:=\frac{\pi \kappa^{\frac{n-2}{2}}}{2(2 \pi)^{\frac{n}{2}}}$, see [18].

\subsection{The uniqueness issue}

The first result of this paper is the following theorem.

Theorem 1.1. (Uniqueness of rough complex obstacles.) Assume that $D_{j}, j=$ 1,2 , are bounded and Lipschitz domains of $\mathbb{R}^{n}$ such that $\mathbb{R}^{n} \backslash \overline{D_{j}}$ is connected and that $\kappa^{2}$ is not a Dirichlet eigenvalue for $(-\Delta)$ on $D_{j}, j=1,2$. Consider two bounded complex valued functions $\lambda_{j}, j=1,2$, defined on $\partial D_{j}, j=1,2$, respectively, with positive real parts. Let $u_{j}^{\infty}(\cdot, \cdot)$ on $\mathbb{S}^{n} \times \mathbb{S}^{n}, j=1,2$, be the far field patterns for the scattering problem (1) - (3) with unknowns $\left(\partial D_{j}, \lambda_{j}\right)$. If $u_{1}^{\infty}(\cdot, \cdot)=u_{2}^{\infty}(\cdot, \cdot)$ on $\mathbb{S}^{n} \times \mathbb{S}^{n}$ then we have $\partial D_{1}=\partial D_{2}$ and $\lambda_{1}=\lambda_{2}$.

There are two ways to prove uniqueness of obstacles from exterior measurements. The first one is due to Schiffer, see for instance [8]. However it is applicable only for Dirichlet type obstacles. The second argument is based on the use of singular solutions by Isakov, Kirsch and Kress, see [16] and [25]. Regarding the uniqueness question, this last argument does not depend on the type of boundary conditions we impose on $\partial D$. Hence it is also 
applicable for the Robin type of boundary conditions. Such arguments are also used for transmission problems, see $[14,15]$. To prove uniqueness of the boundary coefficient $\lambda$, the known and straight argument, see [7] and [25], is to use the boundary conditions coupled with the unique continuation property. However, this last step requires the boundary coefficients to be continuous.

In this paper, we propose another way to prove uniqueness of $(\partial D, \lambda)$ which requires Lipschitz regularity of $\partial D$ and only the bounded-ness of the measurable function $\lambda$. This approach is based on transforming the inverse problem to the invertibility of integral equations involving the unknowns $\partial D$ and $\lambda$. Precisely, we first provide an explicit representation of the Green's function, corresponding to the scattering problem ((1)-(2)-(3)), in terms of the explicit Helmholtz fundamental solution via an invertible integral operator involving $(\partial D, \lambda)$. This shows us how the singular behavior of the Green's function is similar to the one of the fundamental solution. The uniqueness of $\partial D$ is then easy using this representation. We wish to emphasize that this representation is useful to show the weight of $\lambda$ and the geometry of $\partial D$ in the singularity of the Green's function. This is important to understand the power as well as the limits of the known probing and sampling methods, see [20, 21, 27] for some related studies in case of smoother unknowns $(\partial D, \lambda)$. The second integral equation we derive in this paper is of second kind having $\lambda$ as an unknown and its kernel is related to the Green's function of ((1)-(2)-(3)). Its invertibility gives a way how to detect $\lambda$.

\subsection{The reconstruction issue}

The procedure of the proof based on these two integral equations is then reconstructive. Indeed, using the Ikehata-Potthast probe method, for instance ${ }^{1}$, see $[13,24]$, we can compute the values of the Green's function outside the obstacle using the needle approach by Ikehata [13]. The unboundedness of the corresponding indicator functions when the needle approaches the obstacle is justified by the first integral equation we mentioned. Hence, in principle, we get $\partial D$ and the values of the Green's function on $\partial D$. Then inverting the second integral equation we obtain the values of the complex function $\lambda$. This method requires the obstacle to be Lipschitz smooth and the surface impedance to be bounded. Assuming that $\partial D$ to be $C^{2}-$ smooth and $\lambda$ of the class $C^{0, \alpha}(\partial D)$, then using the mentioned representation of the Green's function in terms of the fundamental solution, we show that $\lambda$ can be computed by an exact and stable formula.

1.2.1. Reconstruction of the full Green's function from the far field map. We start by the following key identity

$$
u^{\infty}(\theta, d)=-\gamma_{n} \int_{\partial D}\left\{\frac{\partial u^{s}(y, d)}{\partial \nu} e^{-i \kappa \theta \cdot y}-\frac{\partial e^{-i \kappa \theta \cdot y}}{\partial \nu} u^{s}(y, d)\right\} d s(y)
$$

given by using the Green's formula in $\mathbb{R}^{n} \backslash \bar{D}$ for $u^{s}(\cdot, d)$ and $\Phi(\cdot, y)$ and their asymptotic behavior at infinity (see [8], Theorem 2.5).

By the principle of superposition, we know that, see [8], the scattered field associated with the Herglotz incident field $v_{g}^{i}:=v_{g}$ defined by $v_{g}(x):=\int_{\mathbb{S}^{n}} e^{i \kappa x \cdot d} g(d) d s(d), \quad x \in \mathbb{R}^{n}$, is

\footnotetext{
${ }^{1}$ We could also use another sampling method as the linear sampling method [3] to compute the Green's function of the interior problem instead of the exterior one as for the probe method, see [5]. Hence, we can obtain similar results, as the ones stated in Theorem 1.3, for the linear sampling method.
} 
given by $v_{g}^{s}(x):=\int_{\mathbb{S}^{n}} u^{s}(x, d) g(d) d s(d), \quad x \in \mathbb{R}^{n} \backslash D$, and its far field is given by $v_{g}^{\infty}(\theta):=$ $\int_{\mathbb{S}^{n}} u^{\infty}(\theta, d) g(d) d s(d), \quad \theta \in \mathbb{S}^{n}$.

Let $x, z \in \mathbb{R}^{n} \backslash \bar{D}$. We consider the point sources $\Phi(\cdot, x)$ and $\Phi(\cdot, z)$. We set $B$ such that $\bar{D} \subset B$ and $(x, z) \in \mathbb{R}^{n} \backslash \bar{B}$ and that the Dirichlet interior problem on $B$ is uniquely solvable. In this case, due to the denseness property of the Herglotz wave operator (see [8] or [24]) we take $\left(f_{m}\right)_{m \in \mathbb{N}}$ and $\left(g_{p}\right)_{p \in \mathbb{N}}$ as sequences in $L^{2}\left(\mathbb{S}^{n}\right)$ such that

$$
\left\|v_{f_{m}}-\Phi(\cdot, x)\right\|_{L^{2}(\partial B)} \rightarrow 0, \quad m \rightarrow \infty, \text { and }\left\|v_{g_{p}}-\Phi(\cdot, z)\right\|_{L^{2}(\partial B)} \rightarrow 0, \quad p \rightarrow \infty .
$$

Since $v_{f_{m}}$ and $\Phi(\cdot, x)$ satisfy the same Helmholtz equation in $B$, then by the well posedness of the corresponding interior elliptic problem and the interior estimates, we deduce from (7):

$$
\left\{\begin{array}{l}
\left\|v_{f_{m}}-\Phi(\cdot, x)\right\|_{H^{1}(D)} \rightarrow 0, \quad m \rightarrow \infty \\
\left\|v_{g_{p}}-\Phi(\cdot, z)\right\|_{H^{1}(D)} \rightarrow 0, \quad p \rightarrow \infty .
\end{array}\right.
$$

Multiplying in (6) by $f_{m}(\theta) g_{p}(d)$ and integrating over $\mathbb{S}^{n}$, we obtain:

$$
\begin{aligned}
& \int_{\mathbb{S}^{n}} \int_{\mathbb{S}^{n}} u^{\infty}(-\theta, d) f_{m}(\theta) g_{p}(d) d s(\theta) d s(d) \\
= & -\gamma_{n} \int_{\partial D}\left\{\int_{\mathbb{S}^{n}} \frac{\partial u^{s}(y, d)}{\partial \nu} g_{p}(d) d s(d) \cdot \int_{\mathbb{S}^{n}} e^{i \kappa \theta \cdot y} f_{m}(\theta) d s(\theta)\right. \\
& \left.-\int_{\mathbb{S}^{n}} \frac{\partial e^{i \kappa \theta \cdot y}}{\partial \nu} f_{m}(\theta) d s(\theta) \cdot \int_{\mathbb{S}^{n}} u^{s}(y, d) g_{p}(d) d s(d)\right\} d s(y) \\
= & -\gamma_{n} \int_{\partial D}\left\{\frac{\partial v_{g_{p}}^{s}}{\partial \nu}(y) v_{f_{m}}^{i}(y)-\frac{\partial v_{f_{m}}^{i}}{\partial \nu}(y) v_{g_{p}}^{s}(y)\right\} d s(y) .
\end{aligned}
$$

From (8) and (9), we get:

$$
\begin{aligned}
& \lim _{m \rightarrow \infty} \int_{\mathbb{S}^{n}} \int_{\mathbb{S}^{n}} u^{\infty}(-\theta, d) f_{m}(\theta) g_{p}(d) d s(\theta) d s(d) \\
= & -\gamma_{n} \int_{\partial D}\left\{\frac{\partial v_{g_{p}}^{s}(y)}{\partial \nu} \Phi(y, x)-\frac{\partial \Phi(y, x)}{\partial \nu} v_{g_{p}}^{s}(y)\right\} d s(y) \\
= & \gamma_{n} v_{g_{p}}^{s}(x) .
\end{aligned}
$$

From the well posed-ness of the direct scattering problem and (8), we deduce via interior estimates that

$$
v_{g_{p}}^{s}(x) \rightarrow \Phi^{s}(x, z), \text { when } p \rightarrow \infty .
$$

Hence we proved the following result:

Theorem 1.2. For every $(x, z)$ in $\mathbb{R}^{n} \backslash \bar{D}$, we have

$$
\Phi^{s}(x, z)=\gamma_{n}^{-1} \lim _{p \rightarrow \infty} \lim _{m \rightarrow \infty} \int_{\mathbb{S}^{n}} \int_{\mathbb{S}^{n}} u^{\infty}(-\theta, d) f_{m}(\theta) g_{p}(d) d s(\theta) d s(d),
$$

where $f_{m}$ and $g_{p}$ satisfy ( 7$)$. 
From Theorem 1.2, we compute $G(x, z)$, for every $(x, z)$ in $\mathbb{R}^{n} \backslash \bar{D}$, where $G(\cdot, \cdot)$ is the Green's function associated to the direct scattering problem, i.e

$$
\left\{\begin{array}{l}
\left(\Delta+\kappa^{2}\right) G(x, z)=-\delta(x, z) \text { in } \mathbb{R}^{n} \backslash \bar{D} \\
\frac{\partial G}{\partial \nu_{x}}+i \lambda(x) G=0 \quad \text { if } \quad x \in \partial D \\
G(\cdot, y) \text { satisfies the Radiation conditions }
\end{array}\right.
$$

We call $G$ the Robin function for the Helmholtz scattering problem. In case $\lambda=0$, it is called the Neumann function for the Helmholtz scattering problem and we denote it by $G_{N}$.

From now on, we use the notation $\frac{\partial}{\partial \nu_{x}} G(\cdot, \cdot)$ (resp. $\left.\frac{\partial}{\partial \nu_{z}} G(\cdot, \cdot)\right)$ for the normal derivative with respect to the first (resp. to the second) argument. Arguing as for Theorem 1.2, we can compute the values of $\frac{\partial G}{\partial \nu_{z}}(x, z)$ for $x, z$ in $\mathbb{R}^{n} \backslash \bar{D}$ by choosing $\left(f_{m}\right)_{m \in \mathbb{N}}$ such that $\left\|v_{f_{m}}-\frac{\partial}{\partial \nu_{z}} \Phi(\cdot, x)\right\|_{H^{1}(D)} \rightarrow 0, \quad m \rightarrow \infty$ and $\left(g_{p}\right)_{p \in \mathbb{N}}$ such that $\left\|v_{g_{p}}-\Phi(\cdot, z)\right\|_{H^{1}(D)} \rightarrow 0, \quad p \rightarrow$ $\infty$.

1.2.2. Reconstruction of the complex obstacle The second main theorem of this paper is the following.

Theorem 1.3. (Reconstruction of complex obstacles.) Assume that $D$ is a bounded and a Lipschitz domain of $\mathbb{R}^{n}$ such that $\mathbb{R}^{n} \backslash \bar{D}$ is connected and that $\kappa^{2}$ is not a Dirichlet eigenvalue for $(-\Delta)$ on $D$. Consider a bounded complex valued and measurable function $\lambda$ defined on $\partial D$, with a positive real part. Let $u^{\infty}(\cdot, \cdot)$ on $\mathbb{S}^{n} \times \mathbb{S}^{n}$ be the far field pattern for the scattering problem (1) - (3) with unknowns $(\partial D, \lambda)$. Then

I. The obstacle $D$ can be reconstructed based on the following two properties of the probe method:

I.1. $|I(z)|<\infty$ if $z$ is away from $\partial D$ and

I.2. $\lim _{z \rightarrow \partial D}|I(z)|=\infty$ where

$$
I(z)=\gamma_{n}^{-1} \lim _{p \rightarrow \infty} \lim _{m \rightarrow \infty} \int_{\mathbb{S}^{n}} \int_{\mathbb{S}^{n}} u^{\infty}(-\theta, d) f_{m}(\theta) f_{p}(d) d s(\theta) d s(d),
$$

and the sequence $\left(f_{m}\right)_{m \in N}$ satisfies (7).

II. The complex valued surface impedance $\lambda$ can be reconstructed using one of the two methods

II.1 First method. For this method we need the unknown $\lambda$ to be only a measurable and bounded function with positive real part.

The function $\lambda$ is the solution of the following invertible integral equation:

$$
\lambda(z)-\int_{\partial D} \lambda(x) \frac{\partial G}{\partial \nu_{z}}(x, z) d s(x)=F(z) \text { for } z \in \partial D
$$

from $L^{2}(\partial D)$ to itself, where $F$ is given by

$$
F(z):=-i \int_{\partial D} \frac{\partial G}{\partial \nu_{z}}(x, z)\left[\frac{\partial w}{\partial \nu}(x)\right] d s(x) .
$$

The function $w$ is the solution of the following Dirichlet exterior problem

$$
\left\{\begin{array}{l}
\left(\Delta+\kappa^{2}\right) w=0, \text { in } \mathbb{R}^{n} \backslash \bar{D} \\
w=1, \text { on } \partial D, \\
w \text { satisfies the Sommerfeld radiation conditions. }
\end{array}\right.
$$


II.2 Second method. For this method we assume, in addition, that $\lambda$ is of class $C^{0, \alpha}(\partial D)$, with $\alpha>0$, and $\partial D$ is of class $C^{2}$.

Let $z_{0} \in \partial D$ and denote by $V\left(z_{0}\right)$ an open neighborhood of $z_{0}$ on $\partial D$. Then $\lambda$ is computable on $V\left(z_{0}\right)$ by inverting the following system:

$$
K(z) \lambda(z)=-\int_{\partial D} \frac{\partial}{\partial \nu_{z}} G(x, z) \frac{\partial}{\partial \nu} u_{z}(x) d s(x), z \in V\left(z_{0}\right)
$$

where the complex valued function $K(z)$ is given by

$$
K(z):=i \int_{\partial D} \frac{\partial}{\partial \nu_{z}} G(x, z) \bar{G}(x, z) \xi_{z_{0}}(x) d s(x), \text { for } z \in \overline{V\left(z_{0}\right)}
$$

and for every $z \in \partial D$, the function $u_{z}$ is the solution of the following problem

$$
\left\{\begin{array}{l}
\left(\Delta+\kappa^{2}\right) u_{z}=0, \text { in } \mathbb{R}^{n} \backslash \bar{D} \\
u_{z}=\bar{G}(x, z) \xi_{z_{0}}(x), \text { on } \partial D, \\
u_{z} \text { satisfies the Sommerfeld radiation conditions }
\end{array}\right.
$$

with $\xi_{z_{0}}$ as any $C^{1}$-smooth function defined on $\partial D$ satisfying $\xi_{z_{0}}(x)=0$ for $x \in \overline{V\left(z_{0}\right)}$ and positive elsewhere. In addition, writing (17) as

$$
\left[\begin{array}{cc}
\Re K & -\Im K \\
\Im K & \Re K
\end{array}\right]\left[\begin{array}{l}
\Re \lambda \\
\Im \lambda
\end{array}\right]=\left[\begin{array}{c}
\Re L \\
\Im L
\end{array}\right]
$$

where $L:=-\int_{\partial D} \frac{\partial}{\partial \nu_{z}} G(x, z) \frac{\partial}{\partial \nu} u_{z}(x) d s(x)$, then

$$
\operatorname{det}\left[\begin{array}{cc}
\Re K & -\Im K \\
\Im K & \Re K
\end{array}\right]>0 \text { on } \partial D .
$$

If $\Im \lambda=0$ then $\Re K>0$ on $\partial D$.

For both the methods, we use the following limits:

$$
\left\{\begin{array}{l}
G(x, z)=\lim _{k, h \rightarrow 0} G\left(x_{k}, z_{h}\right), \\
\frac{\partial G}{\partial \nu_{z}}(x, z)=\lim _{k, h \rightarrow 0} \frac{\partial G}{\partial \nu_{z}}\left(x_{k}, z_{h}\right),
\end{array}\right.
$$

in $L^{1}(\partial D \times \partial D)$ where $x_{k}:=x+k \nu(x), z_{h}:=z+h \nu(z)$ and $h, k$ are positive real numbers.

Remark 1.1. 1. Regarding the first method, if we assume, in addition, that $\lambda$ is of class $C^{0, \alpha}(\partial D)$, with $\alpha>0$, and $\partial D$ is of class $C^{2}$, then the integral equation (14) is invertible from $C^{0, \beta}(\partial D)$ to $C^{0, \beta}(\partial D)$ for every $\beta, 0<\beta<\alpha$.

2. Regarding the second method, we can write $\lambda(z)=i \frac{\lim _{k, h \rightarrow 0} \frac{\partial}{\partial \nu} G\left(x_{k}, z_{h}\right)}{\lim _{k, h \rightarrow 0} G\left(x_{k}, z_{h}\right)}$ for $x, z \in \partial D$ by using (22). However, $\lim _{k, h \rightarrow 0} G\left(x_{k}, z_{h}\right)=G(x, z)$ may vanish. To avoid this, we need to fix every point $z$ and then take the limit for $x$ approaching $z$. Similar asymptotic has been used in [19] but it seems that it is quite instable if $\partial D$ is not known with good approximation. This is due to the point-wise behavior with respect to the variable $x$ while the coefficients in $K(z)$ and the second member of (17) are averages with respect to the variable $x$.

Knowing $D$, the straight way of computing $\lambda$ is a direct use of the boundary conditions for the total waves as it is proposed by Akduman and Kress, see [1]. Precisely, for any incident 
wave $u^{i}$, Akduman and Kress propose to compute the corresponding total waves $u$ and $\frac{\partial u}{\partial \nu}$ on $\partial D$ and then solve, by regularization, the equation

$$
u \lambda=i \frac{\partial u}{\partial \nu}
$$

Due to the unique continuation property, the total wave $u$ never vanish in open sets of $\partial D$, however it may vanish on several points of $\partial D$. This is why a regularization is needed in solving (23). An interesting use of $A_{p}$-weights, in case of smooth $(\partial D, \lambda)$, enables Sincich, see [26], to show that $u^{-1}$ is in $L^{p}(\partial D)$ for some values of $p$, which means that (23) is solvable in those $L^{p}$-spaces. As we showed it in Theorem 1.3, part II.2), using superpositions of such incident waves, generating the Green's function, we could transform (23) into (17) which is a stable formula due to $(21)$.

We wish to mention the works by Cakoni, Colton and Monk on the reconstruction of such rough obstacles $(\partial D, \lambda)$, see [3]. Based on the use of the linear sampling method, they reconstruct $D$ and provide an estimate of the $L^{\infty}$-norm of $\lambda$. Assuming that $(\partial D, \lambda)$ are smoother, we showed how we can reconstruct the shape of $D$ and compute the point-wise values of $\lambda$, see [23] and [19] using the probe method, in collaboration with J. Liu and G. Nakamura and [5] using the linear sampling method in collaboration with F. Cakoni, G. Nakamura and N. Zeev, see also [27]. In Theorem 1.3, we improve those results by considering Lipschitz obstacles and reconstructing the surface impedance requiring only its bounded-ness.

The condition that $\kappa^{2}$ is not a Dirichlet eigenvalue for $(-\Delta)$ on $D_{j}, j=1,2$, is due to the use of Layer potentials and it is not a necessary condition for the problem itself.

We wish also to emphasize that the method of the proof proposed here can be used to consider other useful settings as the transmission problems for scalar elliptic operators as well as for Maxwell related models.

The rest of the paper is organized as follows. In section 2, we derive some useful links between the fundamental solution $\Phi$ and the Robing function $G$. In section 3, we use these properties to derive the integral equation we mentioned before having $(\partial D, \lambda)$ as the unknown. In section 4, we use the results of section 2 and section 3 to prove the two stated theorems. Finally, in section 5, we recall some properties of the layer potentials defined by the fundamental solutions and we give a justification of (22).

\section{Some useful links between the Robin function and the funda- mental solution}

We start by the following lemma on the symmetry of the Robin function $G(x, z)$ which proof can be obtained, for instance, as in Lemma 1.9 of [2] by minor changes.

Lemma 2.1. The Robin function $G$ is symmetric in its arguments:

$$
G(x, z)=G(z, x), \text { for } x, z \in \mathbb{R}^{n} \backslash \bar{D} .
$$

Let us recall the single and double layer potentials related to the fundamental solution $\Phi(x, y)$, i.e.

$$
S_{D} f(x):=\int_{\partial D} \Phi(x, z) f(z) d s(z), \text { and } K_{D} g(x):=p \cdot v \cdot \int_{\partial D} \frac{\partial}{\partial \nu_{z}} \Phi(x, z) g(z) d s(z) .
$$


The operators $S_{D}$ and $K_{D}: L^{2}(\partial D) \rightarrow L^{2}(\partial D)$ are bounded, see $[22,10]$. In addition, with respect to the bilinear form $(u, v):=\int_{\partial D} u(x) v(x) d s(x)$, we have $S_{D}^{*}=S_{D}$ and $K_{D}^{*} g=$ $p . v . \int_{\partial D} \frac{\partial}{\partial \nu_{x}} \Phi(x, z) g(z) d s(z)$.

We will use the notation $G_{z}(x):=G(x, z)$. Applying the Green's formula to $G_{z}(x)$ and $\Phi(x, z)$ in $\mathbb{R}^{n} \backslash \bar{D}$, we obtain:

$$
\int_{\partial D} \frac{\partial \Phi}{\partial \nu_{t}}(t, x) G_{z}(t) d s(t)-\int_{\partial D} \frac{\partial G_{z}}{\partial \nu_{t}}(t) \Phi(t, x) d s(t)=G(x, z)-\Phi(x, z), x, z \text { in } \mathbb{R}^{n} \backslash \bar{D} .
$$

Taking the point $x$ to $\partial D$, using the jumps of the double layer potential related to $\Phi$ and the impedance boundary condition for $G_{z}$, we obtain:

$$
\frac{1}{2} G_{z}(x)+K_{D} G_{z}(x)+i S_{D}\left(\lambda G_{z}\right)(x)=G(x, z)-\Phi(x, z), x \text { on } \partial D \text { and } z \text { in } \mathbb{R}^{n} \backslash \bar{D} .
$$

Using the notation $S_{D} \cdot \lambda(f):=S_{D}(\lambda f), \forall f \in L^{2}(\partial D)$, we deduce the following result:

Proposition 2.1. We have the following explicit relation between the Robin function related to the complex obstacle $(\partial D, \lambda)$ and the fundamental solution:

$$
-\left(-\frac{1}{2} I+i S_{D} \cdot \lambda+K_{D}\right) G_{z}(x)=\Phi(x, z), \text { for } x \in \partial D, \text { and } z \in \mathbb{R}^{n} \backslash \bar{D} .
$$

We need the following proposition on the invertibility of the operator $\left(-\frac{1}{2} I+i S_{D} \cdot \lambda+K_{D}\right)$ : $L^{2}(\partial D) \rightarrow L^{2}(\partial D)$ and $\left(-\frac{1}{2} I+i S_{D} \cdot \lambda+K_{D}\right): C^{0, \beta}(\partial D) \rightarrow C^{0, \beta}(\partial D), 0<\beta \leqslant \alpha$.

Proposition 2.2. Suppose that $\partial D$ is Lipschitz and $\lambda \in L^{\infty}$. Assume also that $\kappa^{2}$ is not an eigenvalue for $-\Delta$ in $D$ with Dirichlet boundary conditions. Then

1.) the operator

$$
\left(-\frac{1}{2} I+i S_{D} \cdot \lambda+K_{D}\right): L^{2}(\partial D) \rightarrow L^{2}(\partial D)
$$

is invertible and

2.) if, in addition, $\partial D$ is of class $C^{2}$ and $\lambda \in C^{0, \alpha}(\partial D)$, then the operator

$$
\left(-\frac{1}{2} I+i S_{D} \cdot \lambda+K_{D}\right): C^{0, \beta}(\partial D) \rightarrow C^{0, \beta}(\partial D)
$$

is invertible for every $\beta, 0<\beta \leqslant \alpha$.

Proof of Proposition 2.2

1.) It is enough to prove that its adjoint $-\frac{1}{2} I+i \lambda S_{D}+K_{D}^{*}: L^{2}(\partial D) \rightarrow L^{2}(\partial D)$ is invertible. Indeed, assume that $-\frac{1}{2} I+i \lambda S_{D}+K_{D}^{*}$ is invertible. We set:

$$
-\frac{1}{2} I+i S_{D} \cdot \lambda+K_{D}=: A+P
$$

where $A:=-\frac{1}{2} I+K_{D}$ and $P:=i S_{D} \cdot \lambda$. Since $\kappa^{2}$ is not a Dirichlet eigenvalue then the operator $A$ is invertible from $L^{2}(\partial D) \rightarrow L^{2}(\partial D)$, see the appendix. In addition the operator $P: L^{2}(\partial D) \rightarrow L^{2}(\partial D)$ is compact. Hence we can write:

$$
-\frac{1}{2} I+i S_{D} \cdot \lambda+K_{D}=A\left(I+A^{-1} P\right) .
$$


Now, we write

$$
-\frac{1}{2} I+i \lambda S_{D}+K_{D}^{*}=A^{*}+P^{*}=\left(I+P^{*}\left(A^{*}\right)^{-1}\right) A^{*}
$$

since $A^{*}:=-\frac{1}{2} I+K_{D}^{*}$ is also invertible, as the adjoint of $A$.

Hence the invertibility of $-\frac{1}{2} I+i \lambda S_{D}+K_{D}^{*}$ implies the invertibility of $I+P^{*}\left(A^{*}\right)^{-1}$. Since the operator $P^{*}\left(A^{*}\right)^{-1}$ is compact, we can apply Fredholm alternative to deduce that $I+A^{-1} P$ is also invertible since $I+A^{-1} P=\left(I+P^{*}\left(A^{*}\right)^{-1}\right)^{*}$, see for example [7]. Then $-\frac{1}{2} I+i S_{D} \cdot \lambda+K_{D}$ is invertible.

To show the invertibility of $-\frac{1}{2} I+i \lambda S_{D}+K_{D}^{*}$, we start by recalling that the operator $S_{D}: L^{2}(\partial D) \rightarrow L^{2}(\partial D)$, and then $i \lambda S_{D}$, is compact. Hence the sum $-\frac{1}{2} I+i \lambda S_{D}+K_{D}^{*}$ is Fredholm of index zero. The injectivity of $-\frac{1}{2} I+i \lambda S_{D}+K_{D}^{*}$ in $L^{2}(\partial D)$ is deduced by standard arguments using the uniqueness character of both the exterior impedance problem and the interior Dirichlet problem and the jumps of the double layer potential related to the fundamental solution $\Phi$. Hence the operator $\left(-\frac{1}{2} I+i \lambda S_{D}+K_{D}^{*}\right): L^{2}(\partial D) \rightarrow L^{2}(\partial D)$ is invertible.

2.) Since $\partial D$ is $C^{2}$-smooth then the single layer operator $S_{D}: C^{0, \alpha}(\partial D) \rightarrow C^{1, \beta}(\partial D)$ and the double layer operator $K_{D}: C^{0, \alpha}(\partial D) \rightarrow C^{1, \beta}(\partial D)$ are compact for every $\alpha, \beta>0$ such that $\beta<\alpha$, as combination of bounded operators from $C^{0, \alpha}(\partial D)$ to $C^{1, \alpha}(\partial D)$, see [8], and the compact operator given by the injection of $C^{1, \alpha}(\partial D)$ in $C^{1, \beta}(\partial D)$ for $0<\beta<\alpha$. Now, since $\lambda$ is in $C^{0, \alpha}(\partial D)$, then the operator $-\frac{1}{2} I+i S_{D} \cdot \lambda+K_{D}$ is well defined from $C^{0, \beta}$ to itself, $0<\beta \leqslant \alpha$, and it is a Fredholm operator with index zero. Its injectivity is justified by the previous point 1.). Hence it is invertible.

As a consequence of these last two propositions, we have the following properties of the layer potentials related to the Robin function $G$.

Proposition 2.3. Let $D$ be a Lipschitz domain such that $\mathbb{R}^{n} \backslash \bar{D}$ is connected and $\lambda$ be a complex valued, measurable and bounded function defined on $\partial D$ with a positive real part. Assume that $\kappa^{2}$ is not a Dirichlet eigenvalue of $-\Delta$ in $D^{2}$. Let $G$ be the corresponding Robin function. Then we have the following properties.

1. The single layer potential $\tilde{S}_{G}$ defined by

$$
\tilde{S}_{G} f(z):=\int_{\partial D} G(x, z) f(x) d s(x), \text { with } f \in L^{2}(\partial D)
$$

for $z \in \mathbb{R}^{n} \backslash \bar{D}$, has the trace on $\partial D$ as a bounded operator $S_{G}$ from $L^{2}(\partial D)$ to $L^{2}(\partial D)$ given by

$$
S_{G}=-S_{D}\left(-\frac{1}{2} I+i \lambda S_{D}+K_{D}^{*}\right)^{-1}
$$

In addition, for every $f \in L^{2}(\partial D)$, we have

$$
\lim _{h \rightarrow 0}\left[\tilde{S}_{G} f(z+h \nu(z))-S_{G} f(z)\right]=0 \text { in } L^{2}(\partial D) .
$$

If, in addition, $\partial D$ is of class $C^{2}$ and $\lambda$ is in $C^{0, \alpha}(\partial D)$, then $S_{G}$ is bounded from $C^{0, \beta}(\partial D)$ into $C^{1, \beta}(\partial D)$, for every $\beta, 0<\beta \leqslant \alpha$ and the limit above is understood in the point-wise sense.

\footnotetext{
${ }^{2}$ This condition is needed to use the invertibility of the operator $-\frac{1}{2} I+i \lambda S_{D}+K_{D}^{*}$.
} 
2. The normal derivative of the single layer potential $\tilde{S}_{G} f, \frac{\partial}{\partial \nu} \tilde{S}_{G} f$, has the following trace on $\partial D$ :

$$
-f-i \lambda S_{G} f
$$

In addition, for every $f \in L^{2}(\partial D)$, we have

$$
\lim _{h \rightarrow 0}\left[\frac{\partial}{\partial \nu} \tilde{S}_{G} f(z+h \nu(z))+\left(f(z)+i \lambda(z) S_{G} f(z)\right)\right]=0 \text { in } L^{2}(\partial D) .
$$

The operator $K_{G}^{*}:=-i \lambda S_{G}$ is bounded from $L^{2}(\partial D)$ to $L^{2}(\partial D)$. If, in addition, $\partial D$ is of class $C^{2}$ and $\lambda$ is in $C^{0, \alpha}(\partial D)$, then (32) defines a bounded operator from $C^{0, \beta}(\partial D)$ to $C^{0, \beta}(\partial D)$ and $K_{G}^{*}$ is bounded from $C^{0, \beta}(\partial D)$ to $C^{0, \beta}(\partial D)$, for every $\beta, 0<\beta \leqslant \alpha$ and the limit above is understood in the point-wise sense.

\section{Proof of Proposition 2.3}

1. From Proposition 2.1, we have:

$$
\tilde{S}_{G}=-\tilde{S}_{D}\left(-\frac{1}{2} I+i \lambda S_{D}+K_{D}^{*}\right)^{-1}
$$

where $\tilde{S}_{D} f(z):=\int_{\partial D} \Phi(x, z) f(x) d s(x)$, with $f \in L^{2}(\partial D)$, for $z \in \mathbb{R}^{n} \backslash \bar{D}$.

We set $S_{G}:=-S_{D}\left(-\frac{1}{2} I+i \lambda S_{D}+K_{D}^{*}\right)^{-1}$, hence $S_{G}$ is bounded in the mentioned spaces since $S_{D}$ and $\left.\left(-\frac{1}{2} I+i \lambda S_{D}+K_{D}^{*}\right)^{-1}\right)$ are, see Proposition 2.2 for the latter. The property (31) is a direct consequence of the corresponding one of $S_{D}$.

2. Let $f \in L^{2}(\partial D)$ (or in $C^{0, \beta}(\partial D)$ in case when $(\partial D, \lambda)$ are smoother). We start by taking the derivative in the identity (33) applied to $f$ :

$$
\frac{\partial}{\partial \nu} \tilde{S}_{G} f(z+h \nu(z))=-\frac{\partial}{\partial \nu} \tilde{S}_{D}\left(-\frac{1}{2} I+i \lambda S_{D}+K_{D}^{*}\right)^{-1} f(z+h \nu(z)) .
$$

Using the jumps relation of the normal derivative of the single layer potential in the $L^{2}(\partial D)$ sense (respectively in the point-wise sense for smoother $(\partial D, \lambda)$ ), we obtain

$$
\begin{gathered}
\lim _{h \rightarrow 0} \frac{\partial}{\partial \nu} \tilde{S}_{G}(z+h \nu(z))=-\left[-\frac{1}{2} I+K_{D}^{*}\right]\left(-\frac{1}{2} I+i \lambda S_{D}+K_{D}^{*}\right)^{-1} f(z) \\
=-\left(I-i \lambda S_{D}\left(-\frac{1}{2} I+i \lambda S_{D}+K_{D}^{*}\right)^{-1}\right) f(z) .
\end{gathered}
$$

Hence

$$
\lim _{h \rightarrow 0} \frac{\partial}{\partial \nu} \tilde{S}_{G}(z+h \nu(z))=-f(z)-i \lambda S_{G} f(z)
$$

\section{The reduction to an integral equation}

Let $w$ be the solution of the following Dirichlet scattering problem

$$
\left\{\begin{array}{l}
\left(\Delta+\kappa^{2}\right) w=0, \text { in } \mathbb{R}^{n} \backslash \bar{D} \\
w=1, \text { on } \partial D, \\
w \text { satisfies the Sommerfeld radiation conditions. }
\end{array}\right.
$$

Since $\kappa^{2}$ is not a Dirichlet eigenvalue for $-\Delta$ in $D$, then the operator $S_{D}: L^{2}(\partial D) \rightarrow$ $H^{1}(\partial D)$ is invertible, see the appendix. Let $f \in L^{2}(\partial D)$ such that $S_{D} f=1$. The uniqueness character of Dirichlet exterior problem implies that $w=\tilde{S}_{D} f$. Hence

$$
\left.\frac{\partial w}{\partial \nu}\right|_{\partial D}=S_{D} f-\frac{1}{2} f \in L^{2}(\partial D) .
$$


Integrating by parts using the Green's function $G$, we obtain:

$$
\int_{\partial D} \frac{\partial G}{\partial \nu_{x}}(x, z) d s(x)-\int_{\partial D} \frac{\partial w}{\partial \nu_{x}}(x) G(x, z) d s(x)=w(z), \quad z \in \mathbb{R}^{n} \backslash \bar{D}
$$

which we write as:

$$
\int_{\partial D} \frac{\partial G}{\partial \nu_{x}}(x, z) d s(x)=w(z)+\tilde{S}_{G}\left(\frac{\partial w}{\partial \nu}\right)(z), \text { for } z \in \mathbb{R}^{n} \backslash \bar{D} .
$$

From the impedance boundary condition in (12), we deduce that

$$
-\tilde{S}_{G}(i \lambda)=w(z)+\tilde{S}_{G}\left(\frac{\partial w}{\partial \nu}\right)(z), \text { for } z \in \mathbb{R}^{n} \backslash \bar{D} .
$$

By the trace of the normal derivative of the layer potentials defined by the Robin functions, see Proposition 2.3, we get the following integral equation involving the unknowns $\partial D$ and $\lambda$.

$$
\lambda(z)+\lambda(z) \int_{\partial D} G(x, z)(i \lambda(x)) d s(x)=-i K_{G}^{*}\left(\frac{\partial w}{\partial \nu}\right)(z), \text { for } z \in \partial D
$$

which we can write in a compact form as

$$
\left(I-K_{G}^{*}\right) \lambda=-i K_{G}^{*}\left(\frac{\partial w}{\partial \nu}\right) .
$$

From Theorem 5.2, the kernel of $K_{G}^{*}$, i.e. $-i \lambda(z) G(x, z), x, z \in \partial D$, is computable as

$$
\lim _{k, h \rightarrow 0} \frac{\partial}{\partial \nu_{z}} G\left(x_{k}, z_{h}\right), \text { in } L^{1}(\partial D \times \partial D)
$$

where $x_{k}:=x+k \nu(x)$ and $z_{h}:=z+h \nu(z)$ and $\frac{\partial}{\partial \nu_{z}} G\left(x_{k}, z_{h}\right)$ is computable from the scattering data as in Theorem 1.2 replacing $\Phi(\cdot, x)$ by $\frac{\partial}{\partial x_{j}} \Phi(\cdot, x)$ in $(7)$.

\section{Proofs of Theorem 1.1 and Theorem 1.3}

\subsection{The uniqueness issue (Theorem 1.1)}

The proof of Theorem 1.1 is as follows. Firstly, we recall the known arguments by Isakov, Kirsch and Kress to prove uniqueness of $\partial D$ by adapting them for the rough complex obstacles and using the identity (27). Secondly, knowing $D$ from the first step and $G$ from Theorem 1.2, then using the jumps of the double layer potential corresponding to $G$ and again the identity (27), we show the uniqueness of $\lambda$.

4.1.1. Invertibility of (27) and uniqueness of the obstacles Let $\left(D_{1}, \lambda_{1}\right)$ and $\left(D_{2}, \lambda_{2}\right)$ be two obstacles having the same far field maps $u_{1}^{\infty}(\cdot, \cdot)=u_{2}^{\infty}(\cdot, \cdot)$ on $\mathbb{S}^{n} \times \mathbb{S}^{n}$. Suppose that $D_{1} \neq D_{2}$. We can assume without loss of generality that there exists $\bar{z} \in$ $\partial D_{1} \cap\left(\mathbb{R}^{n} \backslash \overline{D_{2}}\right)$. The function $\left\|\Phi_{z}(\cdot)\right\|_{L^{2}\left(\partial D_{2}\right)}$ is bounded for $z$ near $\bar{z}$, i.e. $z$ is away from $\partial D_{2}$. We set $G_{j}$ to be the Robin function associated to $\left(D_{j}, \lambda_{j}\right), j=1,2$ (as defined in (12)). From the invertibility of the equation (27), we deduce that

$$
\left\|G_{2}(\cdot, z)\right\|_{L^{2}\left(\partial D_{2}\right)} \text { is also bounded for } z \text { near } \bar{z} \text {. }
$$


From the impedance boundary conditions, $\left\|\frac{\partial G_{2}}{\partial \nu}(\cdot, z)\right\|_{L^{2}\left(\partial D_{2}\right)}$ is also bounded for $z$ near $\bar{z}$. Since $G_{2}(\cdot, z)=\Phi_{2}^{s}(\cdot, z)+\Phi(\cdot, z)$ then $\left\|\Phi_{2}^{s}(\cdot, z)\right\|_{L^{2}\left(\partial D_{2}\right)}$ and $\left\|\frac{\partial \Phi_{2}^{s}}{\partial \nu}(\cdot, z)\right\|_{L^{2}\left(\partial D_{2}\right)}$ are also bounded for $z$ near $\bar{z}$.

From Theorem 1.2, we know that $\Phi_{1}^{s}(x, z)=\Phi_{2}^{s}(x, z)$ for $x, z$ in the unbounded connected component of $\mathbb{R}^{n} \backslash\left(D_{1} \cup D_{2}\right)$, which we denote by $D_{1,2}$. Let $\Gamma$ be a part of $\partial D_{1}$ containing $\bar{z}$ and not intersecting $\partial D_{2}$. Without loss of generality, we can assume that $\Gamma \subset \mathbb{R}^{n} \backslash D_{2}$. We set $\Gamma^{c}:=\partial D_{1} \backslash \Gamma$. Then, $\left(\frac{\partial \Phi_{1}^{s}}{\partial \nu_{x}}+i \lambda_{1} \Phi_{1}^{s}\right)(\cdot, z)$ is bounded in $L^{2}(\Gamma)$ for $z$ near $\bar{z}$.

Since $\left(\frac{\partial \Phi_{1}^{s}}{\partial \nu_{x}}+i \lambda_{1} \Phi_{1}^{s}\right)=-\left(\frac{\partial \Phi}{\partial \nu_{x}}+i \lambda_{1} \Phi\right)$ on $\partial D_{1}$, then $\left(\frac{\partial \Phi}{\partial \nu_{x}}+i \lambda_{1} \Phi\right)(\cdot, z)$ is bounded in $L^{2}(\Gamma)$ and hence in $L^{2}\left(\partial D_{1}\right)$, for $z$ near $\bar{z}$. The well posed-ness of the interior problem implies, in particular, that $\|\Phi(\cdot, z)\|_{H^{1}\left(D_{1}\right)}$ is bounded for $z$ near $\bar{z}$, which is impossible. This contradiction implies that $D_{1}=D_{2}$.

4.1.2. Invertibility of (27) and uniqueness of the surface impedances Since the far fields $u_{j}^{\infty}(\cdot, \cdot)$ on $\mathbb{S}^{n} \times \mathbb{S}^{n}, j=1,2$, are equal then from the previous section, we know that $D_{1}=D_{2}$. We set then $D:=D_{1}=D_{2}$. From Theorem 1.2, we have

$$
G_{1}(x, z)=G_{2}(x, z)=: G(x, z), x, z \in \mathbb{R}^{n} \backslash D
$$

and

$$
\nabla_{z} G_{1}(x, z)=\nabla_{z} G_{2}(x, z), x, z \in \mathbb{R}^{n} \backslash \bar{D},
$$

hence by the trace theorem

$$
\frac{\partial G_{1}}{\partial \nu_{z}}(x, z)=\frac{\partial G_{1}}{\partial \nu_{z}}(x, z) \text {, a.e } x \in \partial D \text { and } z \in \mathbb{R}^{n} \backslash \bar{D} .
$$

Let now $f \in L^{2}(\partial D)$, then for $z \in \mathbb{R}^{n} \backslash \bar{D}$, we have

$$
\int_{\partial D} \frac{\partial G_{1}}{\partial \nu_{z}}(x, z) f(x) d s(x)=\int_{\partial D} \frac{\partial G_{2}}{\partial \nu_{z}}(x, z) f(x) d s(x) .
$$

Using the properties of the layer potentials defined by $G$, i.e Theorem 5.2, we obtain:

$$
-f(z)-i \lambda_{1}(z) \int_{\partial D} G_{1}(x, z) f(x) d s(x)=-f(z)-i \lambda_{2}(z) \int_{\partial D} G_{2}(x, z) f(x) d s(x) .
$$

Combining this with (40) gives:

$$
\left(\lambda_{1}-\lambda_{2}\right)(z) \int_{\partial D} G(x, z) f(x) d s(x)=0, z \in \partial D .
$$

The relation

$$
S_{G}=-S_{D}\left(-\frac{1}{2} I+i \lambda S_{D}+K_{D}^{*}\right)^{-1}
$$

the invertibility of $\left(-\frac{1}{2} I+i \lambda S_{D}+K_{D}^{*}\right)$ and the fact that the single layer operator $S_{D}$ has a dense range imply that $S_{G}$ has also a dense range.

From (41) and the denseness of the range of $S_{G}$, we deduce that

$$
\int_{\partial D}\left(\lambda_{1}-\lambda_{2}\right)(z) g(z) d s(z)=0, \forall g \in L^{2}(\partial D) .
$$

This implies that

$$
\lambda_{1}=\lambda_{2}
$$




\subsection{The reconstruction issue (Theorem 1.3)}

In the first part, we justify the two properties of the probe method. If the obstacles $(\partial D, \lambda)$ are smooth, then point-wise estimates of $|\Phi(z, z)|$ can be obtained, see [23] and [24]. We justify this method for Lipschitz obstacles $D$ and complex valued, measurable and bounded surface impedances $\lambda$.

4.2.1. Reconstruction of the obstacle Let $B$ be a $C^{2}$ domain containing the unknown obstacle $D$. We take a point $z_{0} \in \partial B$ and a curve $z:=z(t)_{t \in[0,1]}$ starting from $z_{0}$ and going into the inside of $B$. From the section 2 , we showed how we can reconstruct $\Phi^{s}(z, z)$ from the scattering data. If the curve is out of $D$ then $\left(\frac{\partial}{\partial \nu} \Phi+i \lambda \Phi\right)(\cdot, z)$ is bounded in $L^{2}(\partial D)$. The well posed-ness of the forward scattering problem coupled with the interior estimate imply that $\Phi^{s}(z, z)$ is bounded. Then it is enough to show that when the curve $z$ touches $\partial D, \Phi^{s}(z, z)$ blows up. We proceed as follows. An integration by parts in $B \backslash \bar{D}$, implies that:

$$
\begin{gathered}
\int_{B \backslash \bar{D}}\left|\nabla \Phi^{s}\right|^{2}(x, z)-\kappa^{2}\left|\Phi^{s}\right|^{2}(x, z) d x=\int_{\partial(B \backslash \bar{D})} \frac{\partial \Phi^{s}}{\partial \nu}(x, z) \overline{\Phi^{s}(x, z)} d s(x) \\
=-i \int_{\partial D} \lambda\left|\Phi^{s}\right|^{2}(x, z) d s(x)-\int_{\partial D} \frac{\partial \Phi}{\partial \nu}(x, z) \overline{\Phi^{s}(x, z)} d s(x)-i \int_{\partial D} \lambda \Phi(x, z) \overline{\Phi^{s}(x, z)} d s(x)+O(1)
\end{gathered}
$$

for $z$ near $\partial D$ where we used $O(1)$ as an estimate of the integrals on $\partial B$, since $z$ is far away from $\partial B$.

The Green's formula applied in $B \backslash \bar{D}$ implies again that

$$
\int_{\partial D} \frac{\partial \Phi}{\partial \nu}(x, z) \overline{\Phi^{s}(x, z)} d s(x)=\int_{\partial D} \overline{\frac{\partial \Phi^{s}}{\partial \nu}(x, z)} \Phi(x, z) d s(x)-\overline{\Phi^{s}(z, z)}+O(1) .
$$

Hence

$$
\begin{gathered}
\int_{\partial D} \frac{\partial \Phi}{\partial \nu}(x, z) \overline{\Phi^{s}(x, z)} d s(x)= \\
i \int_{\partial D} \overline{\lambda(x) \Phi^{s}(x, z)} \Phi(x, z) d s(x)-\int_{\partial D} \overline{\frac{\partial \Phi}{\partial \nu}(x, z)} \Phi^{s}(x, z) d s(z)+i \int_{\partial D} \bar{\lambda}|\Phi|^{2}(x, z) d s(x)-\overline{\Phi^{s}(z, z)}+O(1)
\end{gathered}
$$

In addition

$$
\int_{\partial D} \overline{\frac{\partial \Phi}{\partial \nu}(x, z)} \Phi(x, z) d s(x)=\int_{D}|\nabla \Phi|^{2}(x, z)-\kappa^{2}|\Phi|^{2}(x, z) d x .
$$

Using these two last identities in the first equality gives:

$$
\begin{gathered}
\int_{B \backslash \bar{D}}\left|\nabla \Phi^{s}\right|^{2}(x, z)-\kappa^{2}\left|\Phi^{s}\right|(x, z) d x+\int_{D}|\nabla \Phi|^{2}(x, z)-\kappa^{2}|\Phi|^{2}(x, z) d x \\
=-\overline{\Phi^{s}(z, z)}-i \int_{\partial D} \lambda\left|\Phi^{s}\right|^{2}(x, z) d s(x)-i \int_{\partial D} \lambda \Phi(x, z) \overline{\Phi^{s}(x, z)} d s(x) \\
-i \int_{\partial D} \overline{\lambda(x) \Phi^{s}(x, z)} \Phi(x, z) d s(x) i \int_{\partial D} \bar{\lambda}|\Phi|^{2}(x, z) d s(x)+O(1) \text { for } z \text { near } \partial D .
\end{gathered}
$$

Then for $z \in \mathbb{R}^{n} \backslash \bar{D}$, but near $\partial D$, we have

$$
\|\Phi(\cdot, z)\|_{H^{1 / 2}(\partial D)}^{2} \leqslant c\|\Phi(\cdot, z)\|_{H^{1}(D)}^{2} \leqslant c\left[\left|\Phi^{s}(z, z)\right|+\int_{\partial D}\left|\Phi^{s}\right|^{2}(x, z) d s(x)+\int_{\partial D}|\Phi|^{2}(x, z) d s(x)\right.
$$




$$
\left.+\int_{B \backslash \bar{D}}\left|\Phi^{s}\right|^{2}(x, z) d x+\int_{D}|\Phi|^{2}(x, z) d x\right]+O(1) .
$$

However using (27) and Proposition 2.2, we obtain:

$$
\int_{\partial D}\left|\Phi^{s}+\Phi\right|^{2}(x, z) d s(x)=\|G(\cdot, z)\|_{L^{2}(\partial D)}^{2} \leqslant c_{3}\|\Phi(\cdot, z)\|_{L^{2}(\partial D)}^{2}
$$

hence also

$$
\int_{\partial D}\left|\Phi^{s}\right|^{2}(x, z) d s(x) \leqslant \int_{\partial D}\left|\Phi^{s}+\Phi\right|^{2}(x, z) d s(x)+\int_{\partial D}|\Phi|^{2}(x, z) d s(x) \leqslant C\|\Phi(\cdot, z)\|_{L^{2}(\partial D)}^{2}
$$

with $C:=c_{3}+1$. From the explicit form of $\Phi$, we can estimate $\int_{D}|\Phi|^{2}(x, z) d x$. In principle, we have the same for $\Phi^{s}$, and then we can estimate $\int_{B \backslash \bar{D}}\left|\Phi^{s}\right|^{2}(x, z) d x$, since for the Green's function $G$, we have similar estimates as for $\Phi$ as it is shown for a family of scalar equations, see $([12])$ and also $([9])$. Since these references do not include impenetrable obstacles and we are dealing with low regularity for $D$ and the surface impedance $\lambda$, we prefer to provide a justification of a weaker property but enough for our purpose. Indeed, we start by the following identity:

$$
G(x, z)-\Phi(x, z)=\int_{\partial D} \frac{\partial \Phi}{\partial \nu_{t}}(t, x) G_{z}(t)-\int_{\partial D} \frac{\partial G_{z}}{\partial \nu_{t}}(t) \Phi(t, x) d s(t), x, z \text { in } \mathbb{R}^{n} \backslash \bar{D}
$$

which we write as

$$
G(x, z)-\Phi(x, z)=\int_{\partial D}\left[\frac{\partial \Phi}{\partial \nu_{t}}(t, x)-i \lambda(t) \Phi(t, x)\right] G_{z}(t, z) d s(t), x, z \text { in } \mathbb{R}^{n} \backslash \bar{D}
$$

using the impedance boundary conditions. Hence

$$
|G(x, z)-\Phi(x, z)|^{2} \leqslant \int_{\partial D}\left|\frac{\partial \Phi}{\partial \nu_{t}}(t, x)-i \lambda(t) \Phi(t, x)\right|^{2} d s(t) \int_{\partial D}|G(t, z)|^{2} d s(t), x, z \text { in } \mathbb{R}^{n} \backslash \bar{D}
$$

and then

$$
|G(x, z)-\Phi(x, z)|^{2} \leqslant C \int_{\partial D}\left|\frac{\partial \Phi}{\partial \nu_{t}}(t, x)-i \lambda(t) \Phi(t, x)\right|^{2} d s(t) \int_{\partial D}|\Phi(t, z)|^{2} d s(t), x, z \text { in } \mathbb{R}^{n} \backslash \bar{D}
$$

This implies that

$$
\int_{B \backslash \bar{D}}|\Phi(x, z)|^{2} d x \leqslant C\left[\int_{B \backslash \bar{D}}\left|\Phi^{s}(x, z)\right|^{2} d x+\int_{B \backslash \bar{D}} l(x, z) d x\right]
$$

where $l(x, z):=\int_{\partial D}\left|\frac{\partial \Phi}{\partial \nu_{t}}(t, x)-i \lambda(t) \Phi(t, x)\right|^{2} d s(t) \int_{\partial D}|\Phi(t, z)|^{2} d s(t)$.

Finally, from (43), we obtain:

$$
1 \leqslant C\left[\frac{\left|\Phi^{s}(z, z)\right|}{\|\Phi(\cdot, z)\|_{H^{1 / 2}(\partial D)}^{2}}+\frac{\|\Phi(\cdot, z)\|_{L^{2}(\partial D)}^{2}}{\|\Phi(\cdot, z)\|_{H^{1 / 2}(\partial D)}^{2}}+\frac{\int_{B \backslash \bar{D}}\left|\Phi^{s}(x, z)\right|^{2} d x+\int_{B \backslash \bar{D}} l(x, z) d x}{\|\Phi(\cdot, z)\|_{H^{1 / 2}(\partial D)}^{2}}\right] .
$$

Due to the type of singularity of $\Phi$, the second and the third terms of the right hand side of this last inequality are $o(1)$ for $z$ near $\partial D$. Hence, we deduce that there exists a positive constant $\bar{c}$ such that:

$$
\bar{c}\|\Phi(\cdot, z)\|_{H^{1 / 2}(\partial D)}^{2} \leqslant\left|\Phi^{s}(z, z)\right|, \text { for } z \text { near } \partial D
$$

which implies that $\left|\Phi^{s}(z, z)\right|$ blows up for $z$ near $\partial D$. 


\subsubsection{Reconstruction of the surface impedance I) First method}

The unknown surface impedance is solution of the integral equation (37) (or (38)). Knowing the surface of the obstacle $\partial D$, the kernel of the integral operator is constructible in a similar way as in Theorem1.2. Hence the next question is to prove the invertibility of this integral equation.

We start by showing that the operator equation in (38) defined from $L^{2}(\partial D)$ to $L^{2}(\partial D)$ is Fredholm of index zero. We recall that $S_{G}=-S_{D}\left(-\frac{1}{2} I+i \lambda S_{D}+K_{D}^{*}\right)^{-1}$. The operator $S_{G}$ is compact as a product of the bounded operator $\left(-\frac{1}{2} I+i \lambda S_{D}+K_{D}^{*}\right)^{-1}$ and the compact one $S_{D}$. Then $\lambda S_{G}$ is compact too and hence $I+i \lambda S_{G}: L^{2}(\partial D) \rightarrow L^{2}(\partial D)$ is Fredholm of index zero. Now we consider the unique solvability. Suppose that $f+i \lambda \int_{\partial D} G(x, z) f(x) d s(x)=0$, for some $f, f \in L^{2}(\partial D)$. We define $\tilde{S}_{G} f(z):=\int_{\partial D} G(x, z) f(x) d s(x)$ hence it satisfies $\left(\Delta+\kappa^{2}\right) \tilde{S}_{G} f=0$, in $\mathbb{R}^{n} \backslash D$ and the Sommerfeld radiation conditions. In addition, we have $\frac{\partial}{\partial \nu} \tilde{S}_{G} f(z)=-f(z)-i \lambda \int_{\partial D} G(x, z) f(x) d s(x)=0$ on $\partial D$. The uniqueness of the Neumann type scattering problem implies that $\tilde{S}_{G} f(z)=0, z \in \mathbb{R}^{n} \backslash \bar{D}$. Again by the trace property of $\tilde{S}_{G} f$, we deduce that $S_{G} f=0$ and hence $f=0$ using the invertibility of $S_{D}$ and $\left(-\frac{1}{2} I+i \lambda S_{D}+K_{D}^{*}\right)$.

\section{II) Second method}

Let $u_{f}$ be the solution of the problem

$$
\left\{\begin{array}{l}
\left(\Delta+\kappa^{2}\right) u_{f}=0, \text { in } \mathbb{R}^{n} \backslash \bar{D}, \\
u_{f}=f, \text { on } \partial D, \\
u_{f} \text { satisfies the Sommerfeld radiation conditions }
\end{array}\right.
$$

where $f \in H^{1 / 2}(\partial D)$. Since $\partial D$ is $C^{2}$-smooth, then for $f \in C^{1, \beta}(\partial D)$ we associate $u_{f} \in$ $C^{1, \beta}\left(\mathbb{R}^{n} \backslash D\right)$, see $[8]$.

An integration by parts applied for $u_{f}$ and $G(\cdot, \cdot)$ gives:

$$
\int_{\partial D} \frac{\partial}{\partial \nu_{x}} G(x, z) u_{f}(x) d s(x)-\int_{\partial D} \frac{\partial}{\partial \nu_{x}} u_{f}(x) G(x, z) d s(x)=u_{f}(z), z \in \mathbb{R}^{n} \backslash \bar{D}
$$

which we write as:

$$
-i \int_{\partial D} \lambda(x) G(x, z) u_{f}(x) d s(x)=\int_{\partial D} \frac{\partial}{\partial \nu_{x}} u_{f}(x) G(x, z) d s(x)+u_{f}(z), z \in \mathbb{R}^{n} \backslash \bar{D} .
$$

Taking the derivative with respect to $z$ and using the trace of the double layer potential related to $G$, we obtain:

$$
i \lambda(z) f(z)+i \lambda(z) \int_{\partial D} G(x, z)(i \lambda(x)) f(x) d s(x)=-i \lambda(z) \int_{\partial D} \frac{\partial}{\partial \nu_{x}} u_{f}(x) G(x, z) d s(x)
$$

for $z$ in $\partial D$.

We set $\bar{G}(x, z)$ to be the complex conjugate of $G(x, z)$. Let $V\left(z_{0}\right)$ be a neighborhood of $z_{0}$ in $\partial D$. For $x \in \partial D$ and $z \in V\left(z_{0}\right)$, we take $f_{z}(x):=\bar{G}(x, z) \xi_{z_{0}}(x)$, where $\xi_{z_{0}}(x) \geqslant 0$ in $\partial D$ and $\xi_{z_{0}}=0$ for $x \in V\left(z_{0}\right)$. Since $\partial D$ is $C^{2}$-smooth and $\lambda$ is $C^{1, \alpha}(\partial D)$, then the representation $G(\cdot, z)=-\left(-\frac{1}{2} I+i S_{D} \cdot \lambda+K_{D}\right)^{-1} \Phi(\cdot, z)$ and the invertibility of $\left(-\frac{1}{2} I+i S_{D} \cdot \lambda+K_{D}\right)$ from $C^{1, \beta}(\partial D)$ to $C^{1, \beta}(\partial D)$, for every $\beta, 0<\beta \leqslant \alpha$, imply that $G(\cdot, z)$ is $C^{1, \alpha}(\partial D)$. In addition, $f_{z}(x)=0$ for $x, z \in V\left(z_{0}\right)$. Using $f_{z}$ in (48), we have for every $z$ in $V\left(z_{0}\right)$ :

$$
\lambda(z) \int_{\partial D}|G(x, z)|^{2} \xi_{z_{0}}(x) \lambda(x) d s(x)=i \int_{\partial D} \frac{\partial}{\partial \nu_{x}} u_{z}(x) \lambda(z) G(x, z) d s(x),
$$


where we used the notation $u_{z}:=u_{f_{z}}$. We write it as

$$
K \cdot \lambda=L
$$

where

$$
K(z):=\int_{\partial D}|G(x, z)|^{2} \xi_{z_{0}}(x) \lambda(x) d s(x)=i \int_{\partial D}[-i \lambda(x) G(x, z)] \bar{G}(x, z) \xi_{z_{0}}(x) d s(x)
$$

and

$$
L(z):=i \int_{\partial D} \frac{\partial}{\partial \nu_{x}} u_{z}(x) \lambda(z) G(x, z) d s(x) .
$$

From Theorem 5.2, we can compute $G(x, z)$ for $x, z \in \partial D$ as the limit of $G\left(x_{k}, z_{h}\right)$ and $-i \lambda(z) G(x, z)$ as the limit of $\frac{\partial}{\partial \nu_{z}} G\left(x_{k}, z_{h}\right)$, where $x_{k}, z_{h}$ are in $\mathbb{R}^{n} \backslash \bar{D}$. In addition, we can compute $\frac{\partial}{\partial \nu} u_{z}(x)$ by solving the exterior problem (46). Hence $K$ and $L$ are computable from the far field data.

Let us now show that (50) is invertible point-wisely. We write (50) as the following system:

$$
\left[\begin{array}{cc}
\Re K & -\Im K \\
\Im K & \Re K
\end{array}\right]\left[\begin{array}{c}
\Re \lambda \\
\Im \lambda
\end{array}\right]=\left[\begin{array}{c}
\Re L \\
\Im L
\end{array}\right] .
$$

We will show that $\operatorname{det} K>0$ for every point $z \in V\left(z_{0}\right)$. Since this determinant is continuous then there exists a positive constant $c$ such that $\operatorname{det} K \geqslant c$ in $V\left(z_{0}\right)$. We write the determinant as follows

$$
\operatorname{det} K=\left[\int_{\partial D}|G(x, z)|^{2} \xi_{z_{0}}(x) \Re \lambda(x) d s(x)\right]^{2}+\left[\int_{\partial D}|G(x, z)|^{2} \xi_{z_{0}}(x) \Im \lambda(x) d s(x)\right]^{2} .
$$

We assume that $\operatorname{det} K(\bar{z})=0$ for some point $\bar{z}$ in $V\left(z_{0}\right)$. We deduce that $G(x, \bar{z})=0$ for $x \in \partial D \backslash V\left(z_{0}\right)$ since $\xi_{z_{0}}$ and $\Re \lambda$ are positive on $\partial D \backslash V\left(z_{0}\right)$.

If the point $\bar{z}$ was in $\mathbb{R}^{n} \backslash \bar{D}$, then we have also $\frac{\partial}{\partial \nu_{x}} G(x, \bar{z})=0$ for $x \in \partial D \backslash V\left(z_{0}\right)$. By the unique continuation property satisfied by $G(x, \bar{z})$, as a solution of $\left(\Delta+\kappa^{2}\right) G(x, \bar{z})=-\delta(x, \bar{z})$ for $x \in \mathbb{R}^{n} \backslash \bar{D}$, we deduce that $G(x, \bar{z})=0$ for every $x \neq \bar{z}$ which is absurd. However, the point $\bar{z}$ is on $\partial D$ and the argument above is not applicable. For this reason, we proceed as follows. Since $S_{G}\left(-\frac{1}{2} I+i S_{D} \cdot \lambda+K_{D}\right) f=-S_{D} f$ for every $f$ in $L^{2}(\partial D)$, we have

$$
\left(-\frac{1}{2} I+i S_{D} \cdot \lambda+K_{D}\right) G_{z}(x)=-\Phi(x, z), \text { for } x, z \in \partial D .
$$

This means that

$$
\left(i S_{D} \cdot \lambda+K_{D}\right) G_{z}(x)=-\Phi(x, z), \text { for } x \in \partial D \backslash V\left(z_{0}\right) \text { and } z=\bar{z}
$$

We show that this contradicts the singular behavior of the Green's function $G$ and the fundamental solution $\Phi$. Indeed, we use the know estimates $|G(x, z)| \leqslant C_{n}|x-z|^{2-n}$, $|\Phi(x, z)| \leqslant C_{n}|x-z|^{2-n}$ for $n \geqslant 3$ and the log type ones for $n=2$, see [28]. Using these estimates then we obtain

$$
\begin{gathered}
\left|S_{D} \cdot \lambda G_{z}(x)\right| \leqslant\|\lambda\|_{\infty} C_{n}^{2} \int_{\partial D}|x-y|^{2-n}|z-y|^{2-n} d s(y) \sim|x-z|^{3-n} \text { for } n>3 \\
\text { and }\left|S_{D} \cdot \lambda G_{z}(x)\right| \sim \ln (|x-z|) \text { for } n=3
\end{gathered}
$$


In addition, since $\nu$ is of class $C^{1}$ then we have $\left|\frac{\partial \Phi}{\partial \nu}(x, z)\right| \leqslant c_{n}|x-z|^{2-n}$ for $x, z$ on $\partial D$, see for instance [7]. Hence, we have also:

$$
\begin{gathered}
\left|K_{D} G_{z}(x)\right| \leqslant C_{n}^{\prime} \int_{\partial D}|x-y|^{2-n}|z-y|^{2-n} d s(y) \sim|x-z|^{3-n} \text { for } n>3 \\
\text { and }\left|K_{D} G_{z}(x)\right| \sim \ln (|x-z|) \text { for } n=3 .
\end{gathered}
$$

However we know that

$$
|\Phi(x, z)|=C_{n}|x-z|^{2-n}, \text { for } n \geqslant 3 .
$$

This contradicts the equality (54) for $x$ near $V\left(z_{0}\right)$ taking $V\left(z_{0}\right)$ smaller if necessary. A similar contradiction holds for the case $n=2$ using the appropriate singularities. This contradiction implies that $\operatorname{det} K>0$ for every point $z$ on $V\left(z_{0}\right)$.

\section{Appendix}

In the next theorem, we recall some properties of the single layer potentials related to the fundamental solutions $\Phi$.

Theorem 5.1. Let $D$ be a bounded and Lipschitz domain of $\mathbb{R}^{n}$. Assume that $\kappa^{2}$ is not a Dirichlet eigenvalue of $(-\Delta)$ in $D$. The single layer operator

$$
\tilde{S}_{D} f(x):=\int_{\partial D} \Phi(x, z) f(z) d s(z)
$$

defined in $\mathbb{R}^{n} \backslash \partial D$ has a trace on $\partial D$ as a bounded operator $S_{D}$ from $L^{2}(\partial D)$ to $L^{2}(\partial D)$. This operator has the following properties.

1. $S_{D}: L^{2}(\partial D) \rightarrow H^{1}(\partial D)$ is invertible.

2. $S_{D}: L^{2}(\partial D) \rightarrow L^{2}(\partial D)$ has a dense range.

The operator

$$
K_{D} f:=p v \int_{\partial D} \frac{\partial}{\partial \nu_{z}} \Phi(x, z) f(x) d s(x)
$$

and its $L^{2}$ - adjoint

$$
K_{D}^{*} f:=p v \int_{\partial D} \frac{\partial}{\partial \nu_{x}} \Phi(x, z) f(x) d s(x)
$$

have the following properties

3. $\left.\frac{\partial}{\partial \nu} \widetilde{S}_{D} f\right|_{\partial D^{ \pm}}=\left(\mp \frac{1}{2} I+K_{D}\right) f$ for every $f$ in $L^{2}(\partial D)$, where $D^{ \pm}$refers to the trace from outside or inside of $D$.

4. The operators $-\frac{1}{2} I+K_{D}$ and $-\frac{1}{2} I+K_{D}^{*}$ are invertible from $L^{2}(\partial D)$ to $L^{2}(\partial D)$.

\section{Proof of TheOrem 5.1}

We will show the proofs for the points (1) and (4). The proof of the point (2) is equivalent to the proof of the injectivity of the Herglotz wave operator, stated on $\partial D$, which in turn is a consequence of the the fact that $\kappa^{2}$ is not a Dirichlet eigenvalue of $-\Delta$ in $D$, see [8]. The proof of (3) can be found in [22].

For $\kappa=0$, the proofs of the points (1) and (4) are also given, see for instance [10]. The proofs use harmonic analysis. Here, we show that, since $\kappa^{2}$ is not a Dirichlet eigenvalue of $(-\Delta)$ in $D$, then based on the results for the case when $\kappa=0$, we obtain (1) and (4) by a compact perturbation argument and the Fredholm alternative. We give the proofs for the 
dimension greater than 2 . The two dimensional case is similar with appropriate changes due to the form of the corresponding fundamental solutions.

The proof of the point $(\mathbf{1})$. We set $\Phi_{0}(x, z)=\frac{\gamma_{n}}{|x-z|^{n-2}}$. Hence ${ }^{3}$

$$
\Phi(x, z)-\Phi_{0}(x, z)=\gamma_{n} \frac{e^{i \kappa|x-z|}-1}{|x-z|^{n-2}}
$$

and

$$
\nabla \Phi(x, z)-\nabla \Phi_{0}(x, z)=\gamma_{n} \frac{(x-z)}{|x-z|^{n}}\left[i \kappa|x-z| e^{i \kappa|x-z|}-(n-2)\left[e^{i \kappa|x-z|}-1\right]\right] .
$$

In particular, we have

$$
\frac{\partial}{\partial T} \Phi(x, z)-\frac{\partial}{\partial T} \Phi_{0}(x, z)=\gamma_{n} \frac{(x-z) \cdot T}{|x-z|^{n}}\left[i \kappa|x-z| e^{i \kappa|x-z|}-(n-2)\left[e^{i \kappa|x-z|}-1\right]\right]
$$

where $\frac{\partial}{\partial T}$ stands for the tangential derivative.

Let $S_{D}^{0}$ be the single layer potential corresponding to the case $\kappa^{2}=0$. From (55) and (57), we deduce that $S_{D}-S_{D}^{0}$ is compact from $L^{2}(\partial D)$ to $H^{1}(\partial D)$. In particular, $S_{D}$ maps $L^{2}(\partial D)$ to $H^{1}(\partial D)$ since $S_{D}^{0}$ does. In addition, $S_{D}^{0}$ is invertible from $L^{2}(\partial D)$ to $H^{1}(\partial D)$, see $[10]$. Then the operator

$$
\left(S_{D}^{0}\right)^{-1} S_{D}=I+\left(S_{D}^{0}\right)^{-1}\left(S_{D}-S_{D}^{0}\right)
$$

is defined from $L^{2}(\partial D)$ to $L^{2}(\partial D)$ and the operator $\left(S_{D}^{0}\right)^{-1}\left(S_{D}-S_{D}^{0}\right)$ is compact from $L^{2}(\partial D)$ to $L^{2}(\partial D)$. Hence we can apply the Fredholm alternative to $\left(S_{D}^{0}\right)^{-1} S_{D}$. Let $f \in L^{2}(\partial D)$ such that $\left(S_{D}^{0}\right)^{-1} S_{D} f=0$. Then $S_{D} f=0$ and then, since $\kappa^{2}$ is not a Dirichlet eigenvalue, $f=0$. Hence $\left(S_{D}^{0}\right)^{-1} S_{D}$ is invertible. Finally, $S_{D}: L^{2}(\partial D) \rightarrow H^{1}(\partial D)$ is also invertible

The proof of the point (4). It is known that $-\frac{1}{2} I+K_{D}^{0}: L^{2}(\partial D) \rightarrow L^{2}(\partial D)$ is invertible, see [10], where $K_{D}^{0} f:=p v \int_{\partial D} \frac{\partial}{\partial \nu_{z}} \Phi_{0}(x, z) f(x) d s(x)$ for $f \in L^{2}(\partial D)$. From (56), we deduce that the integral operator $K_{D}-K_{D}^{0}$, having $\frac{\partial}{\partial \nu_{z}} \Phi(x, z)-\frac{\partial}{\partial \nu_{z}} \Phi_{0}(x, z)$ as a kernel, is compact from $L^{2}(\partial D)$ to $L^{2}(\partial D)$. We write $-\frac{1}{2} I+K_{D}=\left(-\frac{1}{2} I+K_{D}^{0}\right)\left[I+\left(-\frac{1}{2} I+K_{D}^{0}\right)^{-1}\left(K_{D}-\right.\right.$ $\left.K_{D}^{0}\right)$ ] hence $\left(-\frac{1}{2} I+K_{D}^{0}\right)^{-1}\left(-\frac{1}{2} I+K_{D}\right)=I+\left(-\frac{1}{2} I+K_{D}^{0}\right)^{-1}\left(K_{D}-K_{D}^{0}\right)$. Then we can apply the Fredholm alternative to $\left(-\frac{1}{2} I+K_{D}^{0}\right)^{-1}\left(-\frac{1}{2} I+K_{D}\right)$. The injectivity of $-\frac{1}{2} I+K_{D}$ is due to the fact that $\kappa^{2}$ is not a Dirichlet eigenvalue of $-\Delta$ in $D$ and the jump relations of the single layer potential (point (3)). We conclude that $-\frac{1}{2} I+K_{D}$ is invertible. Its adjoint $-\frac{1}{2} I+K_{D}^{*}$ is also invertible.

In the following theorem, we justify some properties of the Robin function $G$.

Theorem 5.2. Let $D$ be a Lipschitz domain such that $\mathbb{R}^{n} \backslash \bar{D}$ is connected and $\lambda$ be a complex valued, measurable and bounded function defined on $\partial D$ with a positive real part. Assume that $\kappa^{2}$ is not a Dirichlet eigenvalue of $-\Delta$ in $D^{4}$. Let $G$ be the corresponding Robin function. Then we have the following properties:

$$
\left\{\begin{array}{l}
G(x, z)=\lim _{k, h \rightarrow 0} G\left(x_{k}, z_{h}\right) \\
\frac{\partial G}{\partial \nu_{z}}(x, z)=\lim _{k, h \rightarrow 0} \frac{\partial G}{\partial \nu_{z}}\left(x_{k}, z_{h}\right),
\end{array}\right.
$$

in $L^{1}(\partial D \times \partial D)$ where $x_{k}:=x+k \nu(x), z_{h}:=z+h \nu(z)$ and $h, k$ are positive real numbers.

\footnotetext{
${ }^{3}$ For $\kappa=0$ and $n=2$, we take $\Phi_{0}(x, z)=\frac{1}{2 \pi} \ln \left(\frac{1}{|x-z|}\right)$.

${ }^{4}$ This condition is needed to use the invertibility of the representation (27) of Proposition 2.1.
} 
Proof of TheOREM 5.2

Recall that $G_{N}$ is the Neumann function for the exterior problem. The function $(G-$ $\left.G_{N}\right)(\cdot, z), z \in \mathbb{R}^{n} \backslash \bar{D}$ satisfies:

$$
\left\{\begin{array}{l}
\left(\Delta+\kappa^{2}\right)\left(G-G_{N}\right)(x, z)=0 \text { in } \mathbb{R}^{n} \backslash \bar{D} \\
\frac{\partial}{\partial \nu}\left(G-G_{N}\right)=-i \lambda(x) G \text { on } \partial D \\
G(\cdot, z) \text { satisfies the Sommerfeld radiation conditions. }
\end{array}\right.
$$

Hence an integration by parts gives

$$
\left(G-G_{N}\right)(x, z)=-i \int_{\partial D} \lambda(y) G(z, y) G_{N}(x, y) d s(y), \text { for } x, z \in \mathbb{R}^{n} \backslash \bar{D}
$$

and taking the derivative we obtain:

$$
\nabla_{x}\left(G-G_{N}\right)(x, z)=-i \int_{\partial D} \lambda(y) G(z, y) \nabla_{x} G_{N}(x, y) d s(y) \text {, for } x, z \in \mathbb{R}^{n} \backslash \bar{D} .
$$

From the symmetry of $G(x, z)$ and $G_{N}(x, z)$, we have $\nabla_{z} G(x, z)=\nabla_{x} G(z, x)$, and $\nabla_{z} G_{N}(x, z)$ $=\nabla_{x} G_{N}(z, x)$, for $x, z \in \mathbb{R}^{n} \backslash \bar{D}$. Then

$$
\nabla_{z}\left(G-G_{N}\right)(x, z)=\nabla_{x}\left(G-G_{N}\right)(z, x)=-i \int_{\partial D} \lambda(y) G(x, y) \nabla_{x} G_{N}(z, y) d s(y)
$$

For $x$ and $z$ on $\partial D$, we set $x_{k}:=x+k \nu(x)$ and $z_{h}=z+h \nu(z)$, then (61) becomes:

$$
\nabla_{z}\left(G-G_{N}\right)\left(x_{k}, z_{h}\right)=\nabla_{x}\left(G-G_{N}\right)\left(z_{h}, x_{k}\right)=-i \int_{\partial D} \lambda(y) G\left(x_{k}, y\right) \nabla_{x} G_{N}\left(z_{h}, y\right) d s(y) .
$$

The function $x \rightarrow \int_{\partial D} \lambda(z) G(x, z) d s(z)$ is in $L^{2}(\partial D)$ as the image by $S_{G}$ of the $L^{2}(\partial D)$ function $\lambda(z)$. In addition, the function $z \rightarrow \lambda(z) \int_{\partial D} G(x, z) d s(x)$ is also in $L^{2}(\partial D)$. Hence the two integrals $\int_{\partial D}^{x} \int_{\partial D}^{z} \lambda(z) G(x, z) d s(z) d s(x)$ and $\int_{\partial D}^{z} \int_{\partial D}^{x} \lambda(z) G(x, z) d s(x) d s(z)$ make sense and they are equal. Now, we use the following splitting

$$
\begin{gathered}
\int_{\partial D}^{z} \int_{\partial D}^{x} \int_{\partial D}^{y} \lambda(y) G(x+k \nu(x), y) \frac{\partial}{\partial \nu(x)} G_{N}(z+h \nu(z), y) d s(y) d s(x) d s(z) \\
+\int_{\partial D}^{z} \int_{\partial D}^{x} \lambda(z) G(x, z) d s(x) d s(z)= \\
\int_{\partial D}^{z} \int_{\partial D}^{x} \int_{\partial D}^{y} \lambda(y) G(x+k \nu(x), y) \frac{\partial}{\partial \nu(x)} G_{N}(z+h \nu(z), y) d s(y) d s(x) d s(z) \\
-\int_{\partial D}^{z} \int_{\partial D}^{x} \int_{\partial D}^{y} \lambda(y) G(x, y) \frac{\partial}{\partial \nu(x)} G_{N}(z+h \nu(z), y) d s(y) d s(x) d s(z) \\
+\int_{\partial D}^{z} \int_{\partial D}^{x} \int_{\partial D}^{y} \lambda(y) G(x, y) \frac{\partial}{\partial \nu(x)} G_{N}(z+h \nu(z), y) d s(y) d s(x) d s(z) \\
+\int_{\partial D}^{z} \int_{\partial D}^{x} \lambda(z) G(x, z) d s(x) d s(z) .
\end{gathered}
$$

We write the two first integrals of the right hand side of (63) as follows:

$$
\int_{\partial D}^{x} \int_{\partial D}^{y} \lambda(y) G(x+k \nu(x), y) \int_{\partial D}^{z} \frac{\partial}{\partial \nu(x)} G_{N}(z+h \nu(z), y) d s(z) d s(y) d s(x)
$$




$$
-\int_{\partial D}^{x} \int_{\partial D}^{y} \lambda(y) G(x, y) \int_{\partial D}^{z} \frac{\partial}{\partial \nu(x)} G_{N}(z+h \nu(z), y) d s(z) d s(y) d s(x) .
$$

We set $q_{h}(y):=\int_{\partial D}^{z} \frac{\partial}{\partial \nu(x)} G_{N}(z+h \nu(z), y) d s(z)$, for every $h>0$ fixed, then by the limit of the single layer potential related to the Robin function $G$, we obtain:

$$
\int_{\partial D}^{y} \lambda(y) q_{h}(y) G(x+k \nu(x), y) d s(y)-\int_{\partial D}^{y} \lambda(y) q_{h}(y) G(x, y) d s(y) \rightarrow 0 \text { as } k \rightarrow 0
$$

in $L^{2}(\partial D)$ with respect to $x$, which implies that the difference of the two first terms in (63) tend to zero with $k$.

We write now the two last integrals of the right hand side of (63) as follows:

$$
\int_{\partial D}^{z} \int_{\partial D}^{y} \lambda(y) l(y) \frac{\partial}{\partial \nu(x)} G_{N}(z+h \nu(z), y) d s(y) d s(z)+\int_{\partial D}^{z} \lambda(z) l(z) d s(z)
$$

where $l(z):=\int_{\partial D} G(x, z) d s(x) \in L^{2}(\partial D)$. Using the identity $G_{N}=\left(-\frac{1}{2} I+K_{D}^{*}\right)^{-1} \Phi$ and the jumps of the double layer potential related to $\Phi$, we deduce that

$$
\lim _{h \rightarrow 0} \int_{\partial D}^{y} \frac{\partial}{\partial \nu_{x}} G_{N}\left(z_{h}, y\right)(\lambda l)(y) d s(y)=\lambda(z) l(z), \text { in } L^{2}(\partial D) .
$$

Hence, we deduce that (64) goes to zero as $h$ tends to zero.

Summing up, we deduce that the quantity in (63) goes to zero with $k$ and $h$. From (62) and the fact the normal derivative of $G_{N}$ vanishes on $\partial D$, we obtain:

$$
\frac{\partial}{\partial \nu(z)} G\left(x_{k}, z_{h}\right) \rightarrow-i \lambda(z) G(x, z), \text { as } h, k \rightarrow 0, \text { in } L^{1}(\partial D \times \partial D)
$$

We finish the proof by showing that we can exchange the order of the limits with respect to $k$ and $h$. Indeed, from the symmetry of $G$ and $G_{N},(60)$ can be written as

$$
\left(G-G_{N}\right)(x, z)=-i \int_{\partial D} \lambda(y) G(x, y) G_{N}(z, y) d s(y), \text { for } x, z \in \mathbb{R}^{n} \backslash \bar{D}
$$

Hence (62) becomes

$$
\nabla_{z}\left(G-G_{N}\right)\left(x_{k}, z_{h}\right)=i \int_{\partial D} \lambda(y) \nabla_{x} G\left(z_{h}, y\right) G_{N}\left(x_{k}, y\right) d s(y)
$$

Arguing as above, we obtain the same limits but with exchanging the order of the limits of $h$ and $k$.

The proof of limit for $G(x, z)$ is similar.

Acknowledgements. The author thanks Dr. Haibing Wang for having read the manuscript and suggested some improvements on the content and the presentation. He also wishes to thank the referee for his remarks and suggestions. This work is supported by the Austrian Academy of Sciences and the Austrian Science Fund under the project Austrian Science Fund (FWF):[P22341-N18]. 


\section{References}

[1] I. Akduman, R. Kress, Direct and inverse scattering problems for inhomogeneous impedance cylinders of arbitrary shape. Radio Science 38(2003), 1055-1064.

[2] H. Ammari and H. Kang, Polarization and Moment Tensors: with Applications to Inverse Problems and Effective Medium Theory. Applied Mathematical Sciences Series, Volume 162, Springer-Verlag, New York, (2007).

[3] F. Cakoni and D. Colton, Qualitative Methods in Inverse Scattering Theory. An introduction. Interaction of Mechanics and Mathematics. Springer-Verlag, Berlin, (2006).

[4] F. Cakoni, D. Colton and P. Monk, The direct and inverse scattering problems for partially coated obstacles. Inverse Problems 17 (2001), no. 6, 1997-2015.

[5] F. Cakoni; G. Nakamura; M. Sini; N. Zeev, The identification of a penetrable obstacle with mixed transmission conditions from far field measurements. Appl. Anal. 89 (2010), no. 1, 67-86.

[6] R. R. Coifman, M. McIntosh and Y. Meyer, L'intégral de Cauchy définit un opérateur borné su $L^{2}$ pour les courbes Lipschitziennes, Ann. of Math. (2) 116(1982), 361-387.

[7] D. Colton, R. Kress, Integral Equation Methods in Scattering Theory, Pure and Applied Mathematics (New York), A Wiley-Interscience Publication, New York, 1983.

[8] D. Colton and R. Kress, Inverse Acoustic and Electromagnetic Scattering Theory(2nd edition), BerlinSpringer, (1998).

[9] Y. Daido, Gen Nakamura, Reconstruction of inclusions for the inverse boundary value problem with mixed type boundary condition and source term. Inverse Problems 20 (2004), no. 5, 1599-1619.

[10] C. E. Kenig, Harmonic analysis techniques for second order elliptic boundary value problems. CBMS Regional Conference Series in Mathematics, 83. The American Mathematical Society, Providence, RI, 1994. xii+146 pp.

[11] R. Kress, W. Rundell, Inverse scattering for shape and impedance. Inverse Problems 17(2001), 10751085.

[12] M. Grueter, K-O. Widman, The Green function for uniformly elliptic equations, Manuscripta Math, 37, 303-342, (1982).

[13] M. Ikehata, Reconstruction of the shape of the inclusion by boundary measurements, Comm. PDE, Vol.23(1998), 1459-1474.

[14] V. Isakov, On uniqueness of recovery of a discontinuous conductivity coefficient, Comm. Pure Appl. Math., 41 (1988), 865-877.

[15] V. Isakov, On uniqueness in the inverse transmission scattering problem, Comm. Part. Diff. Equat., 15 (1990), 1565-1587.

[16] V. Isakov, Inverse Problems for Partial Differential Equations. Springer Series in Applied Math. Science 127, Berlin: Springer, (1998).

[17] D. S. Jerison and C. Kenig, The Neumann problem in Lipschitz domains, Bull. Amer. Math. Soc., 4 (1981), 203-207.

[18] R. Leis, Initial boundary value problems in mathematical physics. Teubner, Stuttgart 1986.

[19] J.J. Liu, G. Nakamura and M. Sini, Reconstruction of the shape and surface impedance from acoustic scattering data for an arbitrary cylinder. SIAM J. Appl. Math. 67(2007), no. 4, 1124-1146.

[20] J.J. Liu and M. Sini, On the accuracy of reconstruction of obstacles from exterior measurements. Preprint available at http://www.ricam.oeaw.ac.at/people/page/sini/publications.html

[21] J.J. Liu and M. Sini, On the accuracy of the numerical detection of complex obstacles from far field data using the probe method. SIAM J. Sci. Comput., 31(2009), 2665-2687.

[22] W. McLean, Strongly Elliptic Systems and Boundary Integral Equations. Cambridge University press, (2000). 
[23] G. Nakamura and M. Sini, Obstacle and boundary determination from scattering data. SIAM J. Math. Anal. 39 (2007), no. 3, 819-837.

[24] R. Potthast, Point Sources and Multipoles in Inverse Scattering Theory, V 427 of Chapman-Hall/CRC, Research Notes in Mathematics. Chapman-Hall/CRC, Boca Raton, Fl, (2001).

[25] A. Ramm, Inverse Problems, Mathematical and Analytical Techniques with Applications to Engineering, Springer, (2004).

[26] E. Sincich, Stable determination of the surface impedance of an obstacle by far field measurements. SIAM J. Math. Anal. 38 (2006), no. 2, 434-451

[27] N. T. Thanh and M. Sini, An analysis of the accuracy of the linear sampling method for inverse obstacle scattering problems using asymptotic expansion. Inverse Problems 26 (2010), no. 1, 015010, 29 pp.

[28] V. A. Solonnikov, On Green's matrices for elliptic boundary value problems. I Proc. Steklov. Inst. Math, 110 (1970). 